\title{
ANTIARRHYTHMICS BASED ON DITERPENOID ALKALOIDS
}

\author{
M.S. Yunusov, E.M. Tsyrlina, S.A. Kryzhanovsky, I.B. Tsorin, S.G. Yunusova \\ Ufa Institute of Chemistry UFRC Russian Academy of Sciences, \\ Russian Federation, 450054, Ufa, prosp. October, 71,
}

DOI: 10.19163/MedChemRussia2021-2021-133

E-mail:msyunusov@anrb.ru

Diterpenoid alkaloids represent one of the most attractive basic structures for chemical transformations in order to introduce additional pharmacophore groups, since they are available and have a wide range of biological activity antiarrhythmic, local anesthetic, anti-inflammatory, antitumor, etc. In 1987, the hydrobromic salt of lappaconitine was introduced into medical practice as an antiarrhythmic agent called allapinin. Data on antiarrhythmic drugs based on the diterpenoid alkaloid lappaconitine was summarized. The main metabolite of allapinin is $\mathrm{N}$-deacetyllappaconitine (DAL); it significantly surpasses allapinin in the rate of development of the antiarrhythmic effect, which allows to considering it as a potential antiarrhythmic drug that has a significant advantage for injection over allapinin.

A method for the preparation of $\mathrm{N}$-desacetylappaconitine from available lappaconitine is proposed on the basis of the optimization of the acid hydrolysis of lappaconitine. Step-by-step protonation of nitrogen atoms in interaction with strong acids $(\mathrm{HCl}$ and $\mathrm{HBr}$ ) was studied by NMR spectroscopy on $1 \mathrm{H}, 13 \mathrm{C}$, and $15 \mathrm{~N}$ and by potentiometric titration in methanol solutions. The constants and thermodynamic characteristics of DAL protonation in methanol have been determined. The potentiometric method was used to determine the $\mathrm{pH}$ values of $1 \%$ solutions of mono- and di-salts. A method for the preparation of monochlorohydrates and bromohydrates of DAL in crystalline form is proposed. The HPLC method was proposed for the quantitative assessment of the content of monosalts in the injection solution. The efficacy of $\mathrm{DAL} \bullet \mathrm{HCl}$ was studied in a model of cardiac arrhythmias caused by aconitine hydrochloride, calcium hydrochloride and occlusion and subsequent hyperfusion of the coronary artery in anesthetized rats.

A series of works on the synthesis of structural analogs of lappaconitine by transformation of lappaconitine, lappaconine or $\mathrm{N}$-deacetylappaconitine at the aromatic ring, at the amino group of the aromatic ring, or with the participation of the heterocyclic part of the molecule was carried out in order to study their antiarrhythmic properties.

This work was carried out with the financial support of the Russian Science Foundation grant No. 19-13-00096. 